\title{
Antimicrobial resistance in the 21st century
}
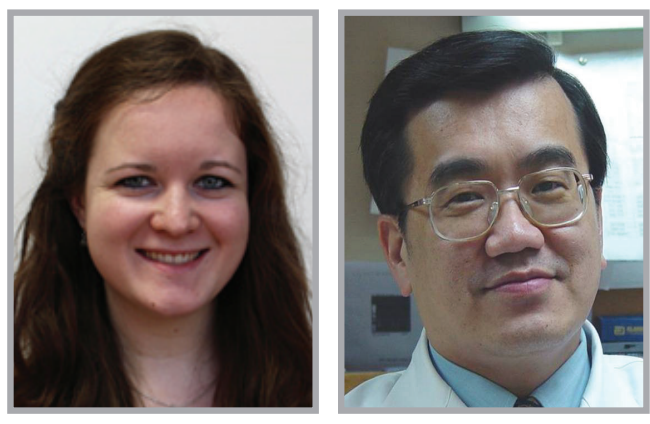

Natasha Leeson ${ }^{* 1}$ \& Po-Ren Hsueh ${ }^{2}$

Antimicrobial resistance (AMR) is a public health problem of increasing magnitude. Although AMR is not a new phenomenon, the speed with which new resistance phenotypes have emerged and the dearth of new agents in the drug development pipeline has elevated the public health significance of this issue. Consequently, we are currently facing a postantibiotic era. In recent years, organizations and governments worldwide have been implementing strategies, working with a range of partners across research, industry and academic sectors, to address this problem [1-4]. AMR is a complex global public health challenge, and no single or simple strategy will suffice to fully contain it. Therefore, to mitigate AMR will be the result of action in the following areas: improving the knowledge and understanding of AMR, conservation of the effectiveness of existing treatments, and developing new antibiotics, diagnostics and novel therapies.

While it is not possible to encapsulate every aspect of AMR in a single special focus issue, Future Microbiology hopes there is something of interest to everyone in this important issue.
In the first of our two thoughtful editorials, Bootsma and Schouls discuss surveillance and infection control of Gram-negative microorganisms resistant to carbapenems, which have become the antibiotics of last resort for many serious bacterial infections [5], while Alsaeed and Blondeau discuss the current standing of antibiotic resistance in hospitals [6]. Whiley et al. follow this by challenging the prospect of untreatable and difficult-to-treat gonorrhea, and highlight the need for more holistic actions to reduce the global gonorrhea burden [7]. Then, Ghafur provides an interesting opinion article on what it was like to be involved in the Chennai Declaration and how this affected the attitude change to AMR in India, emphasizing the global need for action [8].

The next section of our special focus issue includes original research articles, such as Chen et al. who report that overexpression of a novel protein, RfiA, confers resistance to PC190723, a fluoridated benzamide antibiotic recently developed as an inhibitor of the bacterial cell division protein FtsZ [9]. Their work highlights

'Future Medicine Ltd, Unitec House, 2 Albert Place, London, N3 1QB, UK

2Departments of Laboratory Medicine \& Internal Medicine, National Taiwan University Hospital, National Taiwan University College of Medicine, Taipei, Taiwan

*Author for correspondence: Tel.: +44 (0)20 8371 6091; n.leeson@futuremedicine.com 
that the widespread adoption of PC190723 or related benzamides as antimicrobials in clinical practice could lead to resistant pathogens, for example Staphylococcus aureus, eventually acquiring a gene that encodes a member of such novel proteins. Thus, they conclude that these data should inform future policy on careful use of these next-generation antimicrobials to avoid the spread of resistance. Fever and infections represent important complications in patients with cancer, especially during neutropenia following antineoplastic chemotherapy. In an original research article, Castagnola et al. survey resistance to piperacillin-tazobactam, ceftazidime, meropenem and amikacin of Gram-negative rods isolated from bloodstream infections in children with cancer [10]. Based on the epidemiological conditions and resistance profiles, they propose that a combination of an anti-pseudomonal $\beta$-lactam and amikacin appear to be the better choice for initial empirical therapy of children with febrile neutropenia.

In the search for new therapeutic options, Briers and Lavigne review the most recent advances in the development of endolysins as a novel class of antibacterials to treat Gramnegative bacteria [11]. Antimicrobial drugs have been used extensively in livestock and poultry, where entire groups of animals may be treated by the use of medicated feed and water. As a result of continued exposure to antimicrobial drugs, the prevalence of resistant bacteria in the fecal flora of food animals may be relatively high. Determining the impact of these resistant bacteria on human infections is an ongoing challenge as many classes of antimicrobial drugs used in food-producing animals have analogues to human therapeutics and, thus, are capable of selecting for similar resistance phenotypes. Therefore, in a review by Michael et al. the different emerging antimicrobial resistance properties that have been seen in recent years in bacteria of food-producing animals are presented [12].

In conclusion, this special focus issue of Future Microbiology highlights some of the key areas needed to tackle AMR. The increasing collaborative efforts by governments and societies as a whole will generate reliable data as the basis of sound global strategies and public health actions to contain AMR. We look forward to hearing your thoughts on this important topic, and welcome future submissions to Future Microbiology addressing this issue.

\section{Financial \& competing interests disclosure}

$N$ Leeson is an employee of Future Science Group. The authors have no other relevant affiliations or financial involvement with any organization or entity with a financial interest in or financial conflict with the subject matter or materials discussed in the manuscript apart from those disclosed.

No writing assistance was utilized in the production of this manuscript.

\section{References}

1

WHO Antimicrobial resistance: global report on surveillance 2014.

www.who.int

2 Transatlantic Taskforce on Antimicrobial Resistance (TATFAR).

www.cdc.gov

3 Gov.uk Information and resources on the government's plans to slow the growth of antimicrobial resistance.

www.gov.uk

4 Longitude Prize 2014 https://longitudeprize.org/

5 Bootsma HJ, Schouls LM. Next-generation sequencing of carbapenem-resistant
Gram-negative microorganisms: a key tool for surveillance and infection control. Future Microbiol. 10(3), 299-302 (2015).

6 Alsaeed A, Blondeau JM. Antibiotic Resistance in hospitals. Future Microbiol. 10(3), 303-307 (2015)

7 Whiley DM, Lahra MM, Unemo M. Prospects of untreatable gonorrhea and ways forward. Future Microbiol. 10(3), 313-316 (2015).

8 Ghafur A. 'The Chennai Declaration' and the attitude change in India. Future Microbiol. 10(3), 321-323 (2015).

9 Chen X, Zhang B, Xiao J et al. RfiA, a novel PAP2 domain-containing polytopic membrane protein that confers resistance to the FtsZ inhibitor PC190723. Future

Microbiol. 10(3), 325-335 (2015).

10 Castagnola E, Caviglia I, Pescetto L et al. Antibiotic susceptibility of Gram-negatives isolated from bacteremia in children with cancer: implications for empirical therapy of febrile neutropenia. Future Microbiol. 10(3), 357-364 (2015).

11 Briers Y, Lavigne R. Breaking barriers: expansion of the use of endolysins as novel antibacterials against Gram-negative bacteria. Future Microbiol. 10(3), 377-389 (2015).

12 Michael GB, Freitag C, Wendlandt S et al. Emerging issues in antimicrobial resistance of bacteria from food-producing animals. Future Microbiol. 10(3), 427-443 (2015). 